\title{
FIRST RESULTS FROM THE ANGLO-AUSTRALIAN PLANET SEARCH: A BROWN DWARF CANDIDATE AND A 51 PEG-LIKE PLANET ${ }^{1}$
}

\author{
C. G. Tinney, ${ }^{2}$ R. Paul Butler, ${ }^{3,2}$ Geoffrey W. Marcy, ${ }^{4,5}$ Hugh R. A. Jones, ${ }^{6}$ Alan J. Penny, ${ }^{7}$ \\ SteVen S. Vogt, ${ }^{8}$ Kevin Apps, ${ }^{9}$ AND Gregory W. Henry ${ }^{10}$ \\ Received 2000 October 11; accepted 2000 December 3
}

\begin{abstract}
We report results from the Anglo-Australian Planet Search, a survey for planets around 200 solar-type stars in the southern hemisphere that is being carried out on the $3.9 \mathrm{~m}$ Anglo-Australian Telescope. Limiting Doppler precisions of $3 \mathrm{~m} \mathrm{~s}^{-1}$ have been demonstrated from the first 2.5 years of operation, making this the highest-precision planet search in the southern hemisphere. From these data we report results for two new substellar detections. The first is a 51 Peg-like planet around the star HD 179949

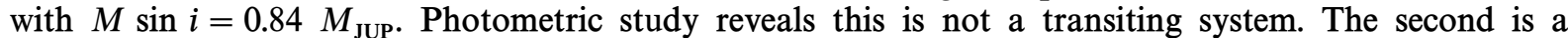
brown dwarf or very low mass star companion to HD 164427 in an eccentric orbit with $M \sin i=46$ $M_{\mathrm{JUP}}$. Hipparcos data indicate this latter object is unlikely to have a mass greater than $0.18 M_{\odot}$. Subject headings: planetary systems — stars: individual (HD 164427, HD 179949) — stars: low-mass, brown dwarfs
\end{abstract}

\section{INTRODUCTION}

Since the discovery of the first extra solar planet by Doppler velocity techniques in 1994 by Mayor \& Queloz (1995), planetary detections have been dominated by northern hemisphere search programs - most prolifically by the precision velocity programs at Lick (e.g., Butler et al. 1996) and Keck (e.g., Vogt et al. 2000), but also complemented by lower precision programs at the Observatoire de HauteProvence (Baranne et al. 1996), McDonald Observatory (Cochran et al. 1997), at Whipple Observatory with the Advanced Fiber Optic Echelle spectrograph (Noyes et al. 1997), and programs at La Silla (Kürster et al. 2000; Queloz et al. 2000). Of these, only the programs at La Silla have access to the sky south of approximately $-20^{\circ}$, and these achieve precisions of $\sim 10 \mathrm{~m} \mathrm{~s}^{-1}$. In 1998, therefore, the Anglo-Australian Planet Search was begun to complete the all-sky coverage of the brightest stars at precisions reaching $3 \mathrm{~m} \mathrm{~s}^{-1}$. In this paper we present some first results from this program. A companion paper (Butler et al. 2001, hereafter Paper II) presents results for a further two new planets, along with a detailed description of our observational program.

\footnotetext{
${ }^{1}$ Based on observations obtained at the Anglo-Australian Telescope, Siding Spring, Australia, and the W. M. Keck Observatory, which is operated jointly by the University of California and the California Institute of Technology.

${ }^{2}$ Anglo-Australian Observatory, P.O. Box 296, Epping 1710, Australia; cgt@aaoepp.aao.gov.au.

${ }^{3}$ Carnegie Institution of Washington, Department of Terrestrial Magnetism, 5241 Branch Road NW, Washington, DC 20015-1305.

${ }^{4}$ Department of Astronomy, University of California, Berkeley, CA 94720.

${ }^{5}$ Department of Physics and Astronomy, San Francisco State University, San Francisco, CA 94132.

${ }^{6}$ Astrophysics Research Institute, Liverpool John Moores University, Twelve Quays House, Egerton Wharf, Birkenhead CH41 1LD, England, UK.

${ }^{7}$ Rutherford Appleton Laboratory, Chilton, Didcot, Oxon OX11 0QX, England, U.K.

${ }^{8}$ UCO/Lick Observatory, University of California, Santa Cruz, CA 95064.

${ }^{9}$ Physics and Astronomy, University of Sussex, Falmer BN1 9QJ, England, U.K.

${ }^{10}$ Center of Excellence in Information Systems, Tennessee State University, Nashville, TN 37203-3401.
}

\section{THE ANGLO-AUSTRALIAN PLANET SEARCH}

The Anglo-Australian Planet Search is being carried out on the $3.92 \mathrm{~m}$ Anglo-Australian Telescope (AAT), using the University College of London Echelle Spectrograph (UCLES) and an $I_{2}$ absorption cell. UCLES is operated in its 31 line $\mathrm{mm}^{-1}$ mode with an MIT/Lincoln Laboratory $2048 \times 409615 \mu \mathrm{m}$ pixel CCD. This lumogen-coated CCD (denoted MITLL2) underwent a serious failure at the end of 1999, which required its readout to be switched to the second of its working amplifiers in 2000 January (following which it was denoted MITLL2a). No change in the operation of the detector (apart from a flipped readout format) has been detected by our program.

Our target sample of $\approx 200$ stars with $\delta<-20^{\circ}$ includes F, G, and K V-IV stars with $V<7.5$ and M V stars with $V<11.5$. Where age/activity information is available from S or $R_{h k}$ indices (see, e.g., Henry et al. 1996), target stars are required to have ages greater than 3 Gyr. Our first observing run was in 1998 January, and the last run for which observations are reported here was in 2000 November. The observing and data processing procedure follows that described in Butler et al. (1996) and is described in detail in Paper II. In particular, Paper II presents velocities for a number of stable stars illustrating that we reach a velocityprecision floor of $3 \mathrm{~m} \mathrm{~s}^{-1}$ for the bright stars included in our program. One of the stars presented here (HD 179949) has also had confirmatory observations acquired at three epochs in 2000 September 5-8, as part of the Keck planet search (Vogt et al. 2000).

\section{CHARACTERISTICS OF HD 179949 AND HD 164427}

HD 179949 (HR 7291, HIP 94645, GJ 749) is an F8 V (Houck \& Smith-Moore 1998) star that shows moderate rotation with a measured $v \sin i=6.3 \pm 0.9 \mathrm{~km} \mathrm{~s}^{-1}$ (Groot, Piters, \& van Paradijs 1996). Its Hipparcos parallax puts it at $27.0 \pm 0.5 \mathrm{pc}$, with $M_{V}=4.09 \pm 0.04$ (ESA 1997). There is no published evidence to indicate that HD 179949 is a binary. The photometry of Eggen (1998) indicates HD 179949 has roughly solar metallicity with $[\mathrm{Fe} / \mathrm{H}]=+0.02 \pm 0.1$. The ubvy calibration of Vogt et al. (2000) and published photometry would indicate a more metal-rich $[\mathrm{Fe} / \mathrm{H}]=+0.22 \pm 0.07$. The latter is 
supported by Twarog \& Anthony-Twarog (1995), Figure 8, which indicates HD 179949 is as metal-rich, if not more so, than the majority of disk main-sequence stars. HD 179949 has a moderate X-ray luminosity as determined by ROSAT's Position Sensitive Proportional CounterHünsch, Schmitt, \& Voges (1998) determine a $\mathrm{L}_{X}=41.0 \times$ $10 \mathrm{ergs} \mathrm{s}^{-1}$, while in an independent analysis Piters et al. (1998) determine $L_{X} / L_{b o l}=(9.7 \pm 3.3) \times 10^{-6}$. Both are some 10 times higher than the equivalent quantity in the quiet Sun. The spectra acquired at Keck have been used to derive a $R_{h k}^{\prime}$ index of -4.72 for this star, using the same procedure as Vogt et al. (2000). Figure 1 compares the $\mathrm{Ca}$ II $\mathrm{H}$ line with that seen in the Sun. The mass of HD 179949 is estimated to be $1.24 \pm 0.1 M_{\odot}$, based on interpolation between evolutionary tracks of Fuhrmann, Pfeiffer, \& Bernkopf (1997) and Fuhrmann, Pfeiffer, \& Bernkopf (1998).

HD 164427 (HIP 88531, Gl 700A) is an inactive G0 V (Houk \& Cowley 1975) star, with a $R_{h k}^{\prime}$ index of -4.95 (Henry et al. 1996). Its Hipparcos parallax puts it at a distance of $39.1 \pm 1.4 \mathrm{pc}$ and $M_{V}=3.91 \pm 0.8$ (ESA 1997), making it somewhat overluminous for its spectral type. Indeed, Evans et al. (1964) classified it as a subgiant, with luminosity class IV. The uvby calibration of Vogt et al. (2000) suggests a metallicity of $[\mathrm{Fe} / \mathrm{H}]=+0.11 \pm 0.07$. HD 164427 was cataloged as a very wide binary (G1 700AB) by Gliese (1969) (although both have since dropped out of the nearby star sample (Gliese \& Jahreiss 1991) because better parallaxes have placed them outside the $25 \mathrm{pc}$ limit). The binary identification is actually due to Luyten (1957), who cataloged this pair as LTT 7172 and LTT 7173, respectively, with common proper motion and a $28^{\prime \prime}$ separation at a position angle of $336^{\circ}$. There is no subsequent astrometry of this pair in the literature. Examination of Digital Sky Survey scans of a United Kingdom Schmidt Telescope plate from 1992.6 shows no evidence for an object with the magnitude difference indicated by Luyten $(\Delta m=7.2)$ at this separation relative to HD 164427 , leading us to conclude it may not be a common proper-motion pair. Even if the system were a binary, it would be a very wide system $(1090$ AU) - so wide as to be irrelevant for the purposes of highprecision Doppler velocities. The mass of HD 164427 is estimated to be $1.05 \pm 0.1 M_{\odot}$.

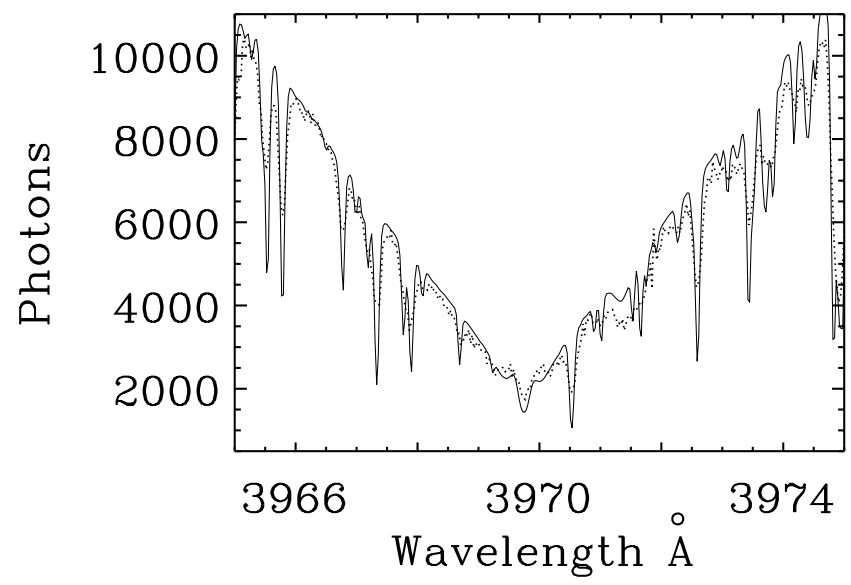

FIG. 1.-Comparison of the Ca II $\mathrm{H}$ line core in the Sun (solid line) and HD 179949 (dotted line). With $R_{h k}^{\prime}=-4.72$ and $v \sin i=6.3 \mathrm{~km} \mathrm{~s}^{-1}$, HD 179949 is slightly more active than the Sun and rotates somewhat faster. The estimated Doppler velocity "jitter" in an F8 V star due to this $\approx 10 \mathrm{~m}$ $\mathrm{s}^{-1}(\operatorname{see} \S 4)$.
Both stars were seen to be photometrically stable over the life of the Hipparcos mission at a $95 \%$ confidence level of $<0.015 \mathrm{mag}$ (ESA 1997).

\section{RADIAL VELOCITY OBSERVATIONS AND ORBITAL SOLUTIONS}

Twenty-three observations of HD 179949 are listed in Table 1, where the column labelled "Uncertainty" is the velocity uncertainty produced by the least-squares fitting process, which simultaneously determines the Doppler shift and the spectrograph point-spread function, given an iodine absorption spectrum, an "iodine-free" template spectrum of the object, and an "iodine" spectrum of the object (Butler et al. 1996). This uncertainty includes the effects of photon-counting uncertainties, residual errors in the spectrograph point-spread function model, and variation in the underlying spectrum between the template and "iodine" epochs. Only observations for which the uncertainty is less than twice the median uncertainty are listed. These data are shown in Figure 2 phased with a period of 3.093 days. The figure shows the best-fit Keplerian model for the data, with the resulting orbital parameters listed in Table 2. The best-fit period from Keplerian fitting is the same as the period found from the peak of the Scargle periodogram, with a false alarm probability less than 0.001 . Both AAT (filled circles) and Keck (squares) data are shown, with the Keck data completely confirming the orbital fits derived from the AAT data alone. Like the previously observed giant planets in 3 to 5 day orbits, HD 179949 appears to be in a circular orbit. Table 2 includes the best-fit orbital solutions both for the case of a circular orbit and floating eccentricity. The resulting minimum companion mass is $0.84 M_{\mathrm{JUP}}$, with an orbital semimajor axis of $0.045 \mathrm{AU}$.

TABLE 1

VELOCITIES FOR HD 179949

\begin{tabular}{lrc}
\hline \hline JD $(-2,440,000)$ & $\begin{array}{r}\text { Radial Velocity } \\
\left(\mathrm{m} \mathrm{s}^{-1}\right)\end{array}$ & $\begin{array}{c}\text { Uncertainty } \\
\left(\mathrm{m} \mathrm{s}^{-1}\right)\end{array}$ \\
\hline $11120.910 \ldots \ldots$. & 118.1 & 5.8 \\
$11383.024 \ldots \ldots$. & -43.3 & 8.9 \\
$11410.943 \ldots \ldots$. & -47.7 & 5.3 \\
$11413.052 \ldots \ldots$. & -15.0 & 4.7 \\
$11413.924 \ldots \ldots$. & -42.1 & 6.1 \\
$11472.917 \ldots \ldots$. & -3.6 & 5.7 \\
$11683.133 \ldots \ldots$. & -40.6 & 8.0 \\
$11684.180 \ldots \ldots$. & 113.7 & 6.1 \\
$11706.151 \ldots \ldots$. & 91.1 & 9.8 \\
$11718.161 \ldots \ldots$. & 127.1 & 6.8 \\
$11742.960 \ldots \ldots$. & 115.9 & 5.9 \\
$11743.992 \ldots \ldots$. & -42.9 & 5.9 \\
$11745.101 \ldots \ldots$. & 0.0 & 5.8 \\
$11766.970 \ldots \ldots$. & 40.8 & 6.2 \\
$11768.000 \ldots \ldots$. & 109.6 & 8.5 \\
$11770.107 \ldots \ldots$. & 44.6 & 6.1 \\
$11792.751^{\mathrm{a}} \ldots \ldots$. & 122.2 & 2.6 \\
$11793.798^{\mathrm{a}} \ldots \ldots$. & -80.7 & 2.5 \\
$11795.803^{\mathrm{a}} \ldots \ldots$. & 115.6 & 2.5 \\
$11827.956 \ldots \ldots$. & -74.3 & 6.1 \\
$11828.911 \ldots \ldots$. & 61.5 & 6.7 \\
$11829.915 \ldots \ldots$. & 117.1 & 8.2 \\
$11855.942 \ldots \ldots$. & -74.4 & 10.3 \\
\hline
\end{tabular}

a Observations from Keck. All other observations are from the AAT. 
TABLE 2

Orbital Parameters

\begin{tabular}{|c|c|c|c|}
\hline Parameter & $\begin{array}{l}\text { HD } 179949 \\
\quad(e=0.0)\end{array}$ & $\begin{array}{l}\text { HD } 179949 \\
(e \text { fitted })\end{array}$ & HD 164427 \\
\hline Orbital period $P$ (days) ............. & $3.093 \pm 0.001$ & $3.093 \pm 0.001$ & $108.55 \pm 0.04$ \\
\hline Velocity amplitude $K\left(\mathrm{~m} \mathrm{~s}^{-1}\right) \ldots \ldots$ & $101.3 \pm 3.0$ & $102.2 \pm 3.0$ & $2229 \pm 77.0$ \\
\hline Eccentricity $e \ldots \ldots \ldots \ldots \ldots \ldots$ & $0.0^{\mathrm{a}}$ & $0.05 \pm 0.03$ & $0.55 \pm 0.02$ \\
\hline 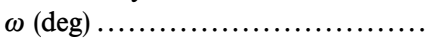 & 0.0 & $226 \pm 25$ & $356.9 \pm 0.5$ \\
\hline 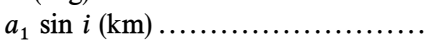 & $4307.3 \pm 15.0$ & $4338.7 \pm 15.0$ & $(2.776 \pm 0.049) \times 10^{6}$ \\
\hline Periastron time (JD-244000) ....... & $11718.19 \pm 0.1$ & $11723.24 \pm 0.1$ & $11724.6 \pm 0.2$ \\
\hline 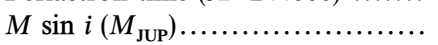 & $0.84 \pm 0.05$ & $0.84 \pm 0.05$ & $46.4 \pm 3.4$ \\
\hline$a(\mathrm{AU}) \ldots \ldots \ldots \ldots \ldots \ldots \ldots \ldots \ldots \ldots \ldots \ldots \ldots \ldots \ldots$ & $0.045 \pm 0.004$ & $0.045 \pm 0.004$ & $0.46 \pm 0.05$ \\
\hline rms about fit $\left(\mathrm{m} \mathrm{s}^{-1}\right) \ldots \ldots \ldots \ldots \ldots$ & 10.8 & 10.3 & 7.7 \\
\hline
\end{tabular}

${ }^{a}$ Fixed.

The residuals about the fit are at the level of "jitter" expected in an F8 V star with HD 179949's level of rotation $\left(6.3 \mathrm{~km} \mathrm{~s}^{-1}\right)$ and activity $\left(R_{h k}^{\prime}=-4.72\right)$. "Jitter" here is used to refer to the scatter in the observed velocity about a

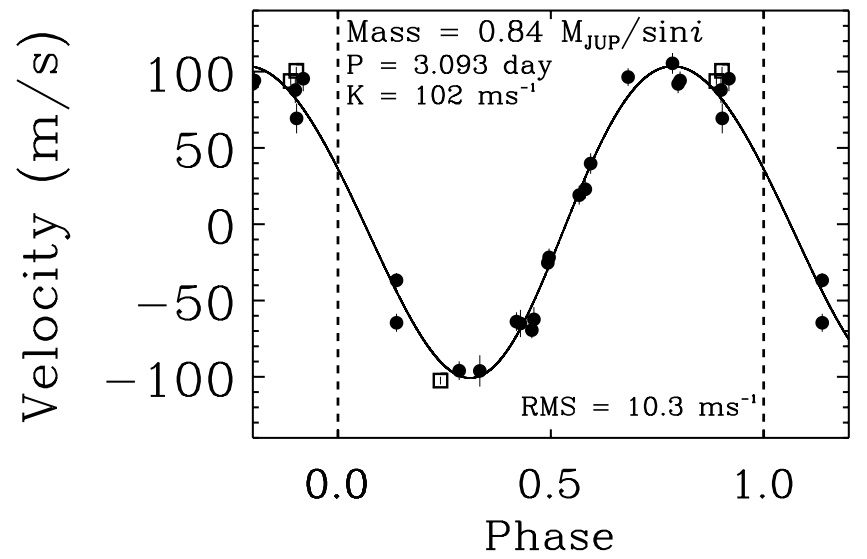

FIG. 2.-AAT ( filled circles) and Keck (squares) Doppler velocities for HD 179949 phased with a period of 3.093 days. The rms of the velocities about the fit is $10.3 \mathrm{~m} \mathrm{~s}^{-1}$. The solid line is a best-fit Keplerian with the parameters shown in Table 2. Assuming 1.24 $\mathrm{M}_{\odot}$ for the primary, the minimum $(M \sin i)$ mass of the companion is $0.84 \pm 0.05 M_{\mathrm{JUP}}$, and the semimajor axis is $0.045 \pm 0.004 \mathrm{AU}$.

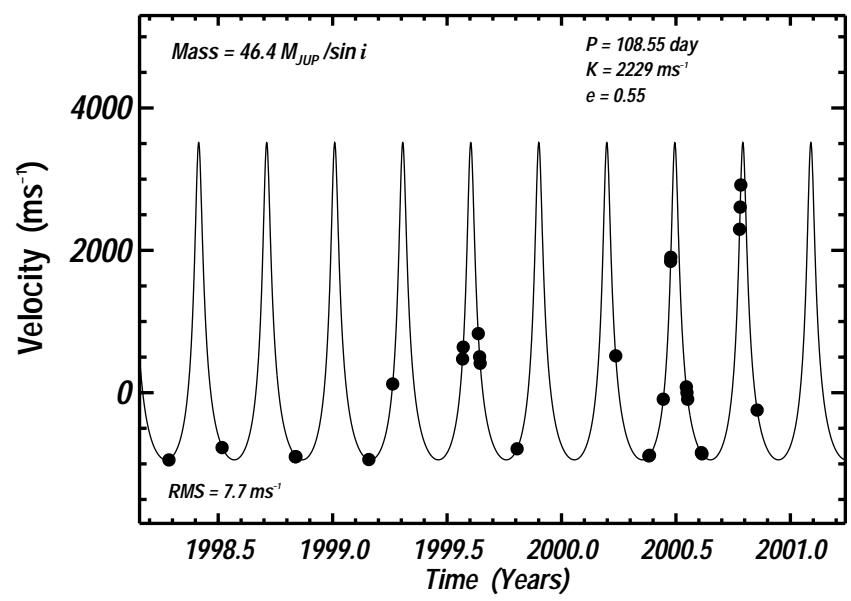

FIG. 3.-AAT Doppler velocities for HD 179949 from 1998 January to 2000 September. The solid line is a best-fit Keplerian with the parameters shown in Table 2. The rms of the velocities about the fit is $7.7 \mathrm{~m} \mathrm{~s}^{-1}$. Assuming a $1.05 M_{\odot}$ for the primary, the minimum $(M \sin i)$ mass of the companion is $46.4 \pm 3.4 M_{\mathrm{JUP}}$, and the semimajor axis is $0.46 \pm 0.05 \mathrm{AU}$. mean value in systems observed over the long term to have no Keplerian Doppler shifts. It is thought to be the combined effect of surface inhomogeneities, stellar activity, and stellar rotation. Saar, Butler, \& Marcy (1998) have studied the correlations between jitter and stellar rotation and activity in the Lick precision velocity program. They indicate we would expect HD 179949 to show $\approx 10 \mathrm{~m} \mathrm{~s}^{-1}$ of jitter due to activity, spots, etc. We measure a scatter of 10 $\mathrm{m} \mathrm{s}^{-1}$ about our Keplerian fit, which is consistent with this expectation.

The 27 observations of HD 166427 are listed in Table 3, and they are shown in Figure 3 along with a Keplerian fit to the data with the orbital parameters listed in Table 2. The rms scatter about this fit is $7.7 \mathrm{~m} \mathrm{~s}^{-1}$, slightly larger than the median uncertainty from the fitting process of $5.6 \mathrm{~m} \mathrm{~s}^{-1}$.

TABLE 3

VELOCITIES FOR HD 164427

\begin{tabular}{|c|c|c|}
\hline $\mathrm{JD}(-2,440,000)$ & $\begin{array}{l}\text { Radial Velocity } \\
\qquad\left(\mathrm{m} \mathrm{s}^{-1}\right)\end{array}$ & $\begin{array}{c}\text { Uncertainty } \\
\left(\mathrm{m} \mathrm{s}^{-1}\right)\end{array}$ \\
\hline $10917.287 \ldots \ldots .$. & -1071.2 & 7.5 \\
\hline $11002.091 \ldots \ldots$. & -897.7 & 10.2 \\
\hline $11118.891 \ldots \ldots$. & -1026.5 & 9.8 \\
\hline $11119.905 \ldots \ldots \ldots$ & -1025.7 & 8.3 \\
\hline $11236.281 \ldots \ldots \ldots$ & -1065.7 & 6.4 \\
\hline $11274.300 \ldots \ldots$. & -3.9 & 5.3 \\
\hline $11385.868 \ldots \ldots$. & 347.2 & 6.8 \\
\hline $11386.879 \ldots \ldots$. & 513.8 & 4.9 \\
\hline $11410.911 \ldots \ldots$. & 703.9 & 4.2 \\
\hline $11413.034 \ldots \ldots$. & 378.0 & 3.6 \\
\hline $11413.904 \ldots \ldots$. & 286.7 & 5.6 \\
\hline $11472.893 \ldots \ldots$. & -914.8 & 4.6 \\
\hline $11630.300 \ldots \ldots$. & 391.3 & 4.9 \\
\hline $11683.096 \ldots \ldots$. & -1014.7 & 5.6 \\
\hline $11684.147 \ldots \ldots$. & -1006.3 & 6.4 \\
\hline $11706.112 \ldots \ldots$. & -217.9 & 4.9 \\
\hline $11717.923 \ldots \ldots$. & 1718.7 & 5.3 \\
\hline $11718.123 \ldots \ldots$. & 1775.8 & 4.9 \\
\hline $11742.913 \ldots \ldots$. & -44.6 & 5.5 \\
\hline $11743.933 \ldots \ldots$. & -126.6 & 7.0 \\
\hline $11745.067 \ldots \ldots$. & -218.7 & 4.6 \\
\hline $11766.942 \ldots \ldots$ & -968.3 & 5.2 \\
\hline $11767.992 \ldots \ldots$. & -985.4 & 8.4 \\
\hline $11827.914 \ldots \ldots$. & 2169.0 & 5.5 \\
\hline $11828.890 \ldots \ldots \ldots$ & 2480.8 & 6.3 \\
\hline $11829.894 \ldots \ldots$. & 2791.5 & 7.0 \\
\hline $11855.921 \ldots \ldots$. & -371.0 & 8.0 \\
\hline
\end{tabular}


This is larger than the systematic precision limit of the Anglo-Australian Planet Search, as the earliest observations of this object were performed in very poor conditions. As soon as it became apparent that HD 164427 was a velocity variable with large amplitude, it was "prorated" within the program to only short exposures (and thus lower precision than the rest of the program), although still more than sufficient to precisely determine the companion's orbit. The resulting minimum companion mass is $46 M_{\mathrm{JUP}}$, or $0.043 M_{\odot}$, and the orbital semimajor axis is $0.46 \mathrm{AU}$.

\section{DISCUSSION}

\subsection{HD 179949}

Ten other 51 Peg-like planets are currently known with periods under 10 days and eccentricities less than $0.1 .^{11}$ The minimum masses of these planets span the range 0.24-3.87 $M_{\mathrm{JUP}}$, with orbital semimajor axes in the range 0.038-0.059 AU. In all respects, HD 179949 falls squarely into this class of objects. Even its above-solar metallicity is similar to that seen in the other 51 Peg-like planets (e.g., Queloz et al. 2000; Gonzalez 2001).

\subsection{Transit Search in HD 179949}

As with all the other 51 Peg-like planets, HD 179949 is an excellent candidate for a transit search. The Hipparcos magnitude, color index, and parallax of the star imply a radius of about $1.24 R_{\odot}$, which, combined with the orbital radius of $0.045 \mathrm{AU}$, places the probability of observable transits at $12.8 \%$. Successful transit observations (already carried out for HD 209458 by Charbonneau et al. 2000 and Henry et al. 2000b) not only tightly constrain orbital inclination, but provide planetary radii as well.

To search for transits in HD 179949, we obtained photometric observations between 2000 September 21 and October 1 with the T8 $0.80 \mathrm{~m}$ Automatic Photoelectric Telescope (APT) at Fairborn Observatory in southern Arizona. ${ }^{12}$ The instrumentation and techniques used for this transit search are described in Henry (1999) and Henry et al. (2000a). The observations were made differentially with respect to the comparison star HD $178075(V=6.30$, $B-V=0.01, \mathrm{~B} 9.5 \mathrm{~V})$. The precision of an individual observation is approximately $0.004 \mathrm{mag}$, somewhat worse than the typical precision of 0.001 mag obtainable with the APT due to observations being at air masses between 2 and 3 .

No transit events were detected at the times predicted by the orbital parameters of Table 2. For an assumed planetary radius of $1.4 R_{\mathrm{JUP}}$ (the measured radius of the planetary companion of HD 209458; Henry et al. 2000b; Jha et al. 2000), the predicted transit depth is 0.014 mag. The mean of our 16 photometric observations of HD 179949 taken within the predicted transit window is $0.1058 \pm 0.0010 \mathrm{mag}$; the mean of the 38 observations outside the transit window is $0.1044 \pm 0.0007$ mag. Thus, the two means agree within their respective errors and transits deeper than about $0.001 \mathrm{mag}$ are ruled out. This nonde-

\footnotetext{
${ }^{11}$ In the absence of a formal classification scheme, we consider HD 83443, HD 168746, HD 46375, HD 75289, BD 10 3166, HD 187123, HD 209458, $v$ And b, and $\tau$ Boo to be 51 Peg-like, as well as 51 Peg itself.

${ }_{12}$ These observations are available at http://schwab.tsuniv.edu/t8/ hd179949/hd179949.html.
}

tection of transits limits the orbital inclination $i$ to less than $83^{\circ}$, and $\sin i$ to less than 0.992 . Furthermore, the photometric observations place a limit of about $0.001 \mathrm{mag}$ on any photometric variability of HD 179949 on the radial velocity period. This confirms that neither starspots nor stellar pulsations can be the cause of the radial velocity variations (see Henry et al. 2000a), and thus strongly supports the existence of the planet even in the absence of transits.

\subsection{HD 164427}

Recent work on the mass function of brown dwarfs in star clusters (Luhman et al. 2000; Lucas \& Roche 2000; Zapatero Osorio et al. 2000) has failed to show that star formation processes cannot form objects below the $13 M_{\text {JUP }}$ deuterium-burning limit. (Although the same results can be interpreted - less straightforwardly - as implying the existence of free-floating sub-13 $M_{\mathrm{JUP}}$ objects formed by dynamical evolution ejecting objects formed by planetary processes.) Certainly they provide no evidence that the deuterium-burning limit is a useful demarcation boundary between star and planet formation processes. Difficult as it may be, only an understanding of formation mechanisms (and possible subsequent dynamical evolution) can allow us to determine whether objects in the 5-20 $M_{\mathrm{JUP}}$ range are planets or brown dwarfs. Nonetheless, the minimum mass of the companion to HD 164427 clearly lies in a range unlikely to be due to planetary formation processes - that is, it is a brown dwarf or a star, not a planet.

Hipparcos astrometry, however, allows us to place some limits on the orbital inclination and mass for HD 164427's companion. The astrometric solution for HD 164427 shows a good fit for the parallax and proper motion of a single star, with $1 \sigma$ residuals of $\approx 3$ mas. The uncertainty on the derived parallax is 0.9 mas. Based on this, we can assume an upper limit to the astrometric perturbation $\left(a_{0}\right)$ of HD 164427 due to an unseen companion of $\approx 2$ mas. Then, from the relationship between the spectroscopic and astrometric orbital elements (e.g., Halbwachs et al. 2000, eq. [1]), we conclude that $\sin i>0.24$, or equivalently that the companion mass is less than $190 M_{\mathrm{JUP}}$ or $0.18 M_{\odot}$, making it either a brown dwarf or a very low mass star. With an orbital semimajor axis of $0.46 \mathrm{AU}$ at $39 \mathrm{pc}$, such a companion will have a maximum apparent separation of 11.8 mas. Table 4 compares the absolute $K$ magnitudes of HD 164427 with predicted magnitudes of the companion in the range of allowed masses. Only at the very massive end of this range is the companion likely to be detectable by either groundbased adaptive optics or space-based imaging.

TABLE 4

Predicted $K$ MAGNitUdes FOR HD 164427 COMPANION $M_{K}=5.4$

\begin{tabular}{cccc}
\hline \hline Companion Mass & Age $(\mathrm{Gyr})$ & $M_{\boldsymbol{K}}$ & $\Delta M_{\boldsymbol{K}}$ \\
\hline $0.18 M_{\odot}{ }^{\mathrm{a}} \ldots \ldots \ldots$ & $\ldots$ & 7.7 & 2.3 \\
$0.10 M_{\odot}^{\mathrm{a}} \ldots \ldots \ldots$ & $\ldots$ & 9.2 & 3.8 \\
& 1 & 11.2 & 5.9 \\
$75 M_{\mathrm{JUP}}{ }^{\mathrm{b}} \ldots \ldots \ldots$. & 5 & 11.7 & 6.3 \\
$42 M_{\text {JUP }}{ }^{\mathrm{b}} \ldots \ldots \ldots \ldots$ & 1 & 13.7 & 8.3 \\
\hline
\end{tabular}

${ }^{\text {a }}$ Empirical $M_{K}$ values due to Henry \& McCarthy (1993).

${ }^{b}$ Model $M_{K}$ values due to Chabrier et al. (2000). 
Nonetheless, such a challenging observation must be pursued. Of the small number of brown dwarf candidates identified by precision Doppler surveys to date (around 11), many have been shown to actually be stars (Halbwachs et al. 2000), making bona fide brown dwarf companions rare and well worth unambiguously identifying.

\section{CONCLUSIONS}

We present first results from the Anglo-Australian Planet Search, which is now the highest-precision Doppler planet search in the southern hemisphere. These include a new member of the 51 Peg-like class of planets around the F8 dwarf HD 179949 and a new brown dwarf candidate companion to the G0 dwarf HD 164427. This program has now been running on the AAT for over 2 years and has demonstrated velocity precisions reaching $3 \mathrm{~m} \mathrm{~s}^{-1}$. We confidently expect many more discoveries in the years to come- particularly as it extends its sensitivity toward the Jupiterlike planet regime.

The Anglo-Australian Planet Search team would like to gratefully acknowledge the support of the Director of the AAO, Dr. Brian Boyle, and the superb technical support provided throughout the program by AAT staff-in particular E. Penny, R. Patterson, D. Stafford, F. Freeman, S. Lee, J. Pogson, and G. Schafer. We further acknowledge support by the partners of the Anglo-Australian Telescope Agreement (C. G. T., H. R. A. J., A. J. P.); NASA grant NAG 5-8299 and NSF grant AST 95-20443 (G. W. M.); NASA grant NAG 5-4445 and NSF grant AST 96-19418 (S. S. V.); NSF grant AST 99-88087 (R. P. B.); NASA grants NCC5-96 and NCC5-228, NSF grant HRD 97-06268, and the Richard Lounsbery Foundation (G. W. H.); and Sun Microsystems.

\section{REFERENCES}

Baranne, A., et al. 1996, A\&AS, 119, 373

Butler, R. P., Marcy, G. W., Williams, E., McCarthy, C., \& Dosanjh, P. 1996, PASP, 108, 500

Butler, R. P., Tinney, C. G., Marcy, G. W., Jones, H. R. A., Penny, A. J., Apps, K. 2001, ApJ, in press

Chabrier, G., Baraffe, I., Allard, F., \& Hauschildt, P. 2000, ApJ, 542, 464

Charbonneau, D., Brown, T. M., Latham, D. W., \& Mayor, M. 2000, ApJ, $529, \mathrm{~L} 45$

Cochran, W. D., Hatzes, A. P., Butler, R. P., \& Marcy, G. W. 1997, ApJ, 483, 457

Eggen, O. J. 1998, AJ, 115, 2397

European Space Agency 1997, The Hipparcos and Tycho Catalogues (SP1200) (Noordwijk: ESA)

Evans, D. S., Laing, J. D., Menzies, A., \& Stoy, R. H. 1964, R. Obs. Bull., 85, 207

Fuhrmann, K., Pfeiffer, M. J., \& Bernkopf, J. 1997, A\&A, 326, 1081 1998, A\&A, 336, 942

Gliese, W. 1969, Catalogue of Nearby Stars (Karlsruhe: G. Braun)

Gliese, W., \& Jahreiss, H. 1991, Preliminary Version of the Third Catalogue of Nearby Stars (Heidelberg: Astron. Rechen-Inst.)

Gonzalez, G., Laws, C., Tyagi, S., \& Reddy, B. E. 2001, AJ, 121, 432

Groot, P. J., Piters, A. J. M., \& van Paradijs, J. 1996, A\&AS, 118, 545

Halbwachs, J. L., Arenou, F., Mayor, M., Udry, S., \& Queloz, D. 2000, A\&A, 355, 581

Henry, G. W. 1999, PASP, 111, 845

Henry, G. W., Baliunas, S. L., Donahue, R. A., Fekel, F. C., \& Soon, W. 2000a, ApJ, 531, 415

Henry, G. W., Marcy, G. W., Butler, R. P., \& Vogt, S. S. 2000b, ApJ, 529, L41

Henry, T. J., \& McCarthy, D. W., Jr. 1993, AJ, 106, 773

Henry, T. J., Soderblom, D. R., Donahue, R. A., \& Baliunas, S. L. 1996, AJ, 111,439
Houk, N., \& Cowley, A. P. 1975, Michigan Catalogue of Two-Dimensional Spectral Types for the HD stars, Vol. 1 (Ann Arbor: Univ. Michigan)

Houk, N., \& Smith-Moore, M. 1988, Michigan Catalogue of TwoDimensional Spectral Types for the HD stars, Vol. 4 (Ann Arbor: Univ. Michigan)

Hünsch, M., Schmitt, J. H. M. M., \& Voges, W. 1998, A\&AS, 132, 155

Jha, S., Charbonneau, D., Garnavich, P. M., Sullivan, D. J., Sullivan, T., Brown, T. M., \& Tonry, J. L. 2000, ApJ, 540, L45

Kürster, M., Endl, M., Els, S., Hatzes, A. P., Cochran, W. D., Döbereiner, S., \& Dennerl, K. 2000, A\&A, 353, L33

Lucas, P. W., \& Roche, P. F. 2000, MNRAS, 314, 858

Luhman, K. L., Rieke, G. H., Young, E. T., Cotera, A. S., Chen, H., Rieke, M. J., Schneider, G., \& Thompson, R. I. 2000, ApJ, 540, 1016

Luyten, W. J. 1957, A Catalogue of 9867 Stars in the Southern Hemisphere with Proper Motions Exceeding 0.2" Annually (Minneapolis: Lund)

Mayor, M., \& Queloz, D. 1995, Nature, 378, 355

Noyes, R., Jha, S., Korzennik, S., Krockenberger, M., Nisenson, P., Brown, T., Kennelly, E., \& Horner, S. 1997, in ASP Conf. Ser. 119, Planets beyond the Solar System and the Next Generation of Space Missions, ed. D. Soderblom (San Francisco: ASP), 119

Piters, A. J. M., van Paradijs, J., \& Schmitt, J. H. M. M. 1998, A\&AS, 128, 29

Queloz, D., et al., 2000, A\&A, 354, 99

Saar, S. H., Butler, R. P., \& Marcy, G. W. 1998, ApJ, 498, L153

Twarog, B. A., \& Anthony-Twarog, B. J. 1995, AJ, 109, 2828

Vogt, S. S., Marcy, G. W., Butler, R. P., \& Apps, K. 2000, ApJ, 536, 902

Zapatero Osorio, M. R., Béjar, V. J. S, Martín, E. L., Rebolo, R., Barrado y Navascués, D., Bailer-Jones, C. A., \& Mundt, R. 2000, Science, 290, in press 